ISSN: 2602-8506

Vol. 3, N4., p.98-115, octubre - diciembre, 2019

Recibido: 04-08-2019 /Aceptado:08-09-2019/ Publicado: 05-10-2019

\title{
La responsabilidad social corporativa y el clima laboral
}

\section{Corporate social responsibility and the work environment}

Washington Edy Santillán Marroquín. ${ }^{1}$, Hernán Eduardo Paredes García. ${ }^{2}$, Héctor Patricio Mera Manotoa. ${ }^{3}$ \& Josué Fernando Santillán Vieira. ${ }^{4}$

\section{DOI: https://doi.org/10.33262/visionariodigital.v3i4.974}

\begin{abstract}
Explained by the technical measurement of the working environment, the importance of the Corporate Social responsibility (CSR) in the organizations and their influence on the overall wellbeing of staff, through a practical exercise, using a survey of measurement of working environment, where was assessed quantitatively and qualitatively some critical factors for the company, to verify that an excellent working climate has a positive influence on CSR. CSR, not only focuses on the external environment of the company, also refers to the internal responsibility having this company and that is related to its partners in the management of human talent, especially in the treatment and relationship benefits granted; as well as the responsibility that each individual, as collaborator and that should be in every act already is in the role of leader, administrator, employee, customer or supplier of the company.
\end{abstract}

Keywords: Responsibility, paradigm, social approach, labor, solidarity, ethical climate of management, human resources, relationship, benefits, customer, supplier.

\section{Resumen}

Explicar mediante la medición técnica del clima laboral, la importancia de la Responsabilidad Social Corporativa (RSC) en las Organizaciones y su influencia en el bienestar integral del personal, mediante un ejercicio práctico, utilizando una encuesta de medición de clima laboral, en donde se valoró cuantitativamente y cualitativamente

\footnotetext{
${ }^{1}$ Coordinador académico, Quito, Ecuador, coordinacion.academica@instituto-ohiggins.com

${ }^{2}$ Rector Instituto Superior Tecnológico Bernardo Ohiggins, Quito, rectorado@instituto-ohiggins.com

${ }^{3}$ Docente de Administración. Quito, patomera@ hotmail.com

${ }^{4}$ Maestrante de Talento Humano, Quito, josue.f.santillana.viera@ec.ey.com
} 
algunos factores críticos para la compañía, para comprobar que un excelente clima laboral influye positivamente en la RSC.

La RSC, no solo se centra en el ámbito externo de la Empresa, también se refiere a la responsabilidad interna que tiene dicha Compañía y que se relaciona con sus colaboradores en la gestión del talento humano, especialmente en el trato, relación y beneficios otorgados; así como, la responsabilidad que cada individuo, como colaborador y que debe tener en cada acto ya sea desde el rol de líder, administrador, trabajador, cliente o proveedor de la empresa.

Palabras claves: Responsabilidad, paradigma, enfoque social, clima laboral, solidario, ético de la gestión, talento humano, relación, beneficios, cliente, proveedor.

\section{Introducción}

Se vuelve relevante este tema al considerar que la Responsabilidad Social Corporativa, no solo es realizar una serie de prácticas profesionales y bien intencionadas con el entorno y la comunidad en donde funciona la Organización, si no, que los colaboradores de una organización se convierten en interlocutores válidos de la empresa; es decir, en su gestión profesional debe tomarse en cuenta las acciones prácticas y permanentes para generar un "un buen ambiente laboral" así como, la existencia de un diálogo sostenido con todos los niveles de la empresa, bajo principios de valores, sinceridad, inclusión de todos los protagonistas, reciprocidad y simétricos para buscar acuerdos entre las partes. Por este motivo las organizaciones se las considera instituciones sociales, en donde sus intereses, acciones y decisiones son susceptibles de valor moral, ético y espiritual.

El enfoque de la Responsabilidad Social Corporativa ha despertado un enorme interés en el sector empresarial, las escuelas de negocio, los inversores e incluso algunos líderes. A este interés se le suma la influencia que ejerce la Gestión Humana en las organizaciones, al facilitar y generar competencias específicas para la empresa, y combinar a los grupos sociales para imprimir mayor eficacia en el logro de los objetivos organizacionales y mejoramiento continuo del clima laboral.

La RSC es un enfoque vigente en la gestión actual de las organizaciones, con una gran variedad de definiciones atribuidas. Proviene de una tradición norteamericana y británica a principios del siglo XX, aunque para algunos autores como Carroll (1999), el concepto solo aparece hasta 
ISSN: 2602-8506

Vol. 3, N $4 .$, p.98-115, octubre - diciembre, 2019

mediados del mismo siglo con la publicación del libro Social Responsibilities of The Businessman, de Howard Bowen, en 1953.

La RSC es considerada como un enfoque de gestión estratégico generador de oportunidades, innovación y ventaja competitiva para las organizaciones, con una repercusión en su ambiente, imagen y reputación.

Objetivo General: Crear un ambiente interno y externo adecuado, para que se considere un "gran sitio para trabajar", mediante el buen trato y equidad, donde se vea reflejado el compromiso de todos sus actores, con la finalidad de generar mayor productividad y rentabilidad, sin olvidar el compromiso de minimizar cualquier tipo de contaminación ambiental.

\section{Objetivos Estratégicos:}

- Alcanzar el interés social de la empresa, mejoramiento continuo del clima laboral y logro de sus objetivos estratégicos.

- Gestionar la empresa desde los valores de solvencia, integridad, vocación de servicio, innovación para el liderazgo y los equipos de trabajo.

- Establecer relaciones estables y de equidad con los grupos de interés con los que se relaciona la empresa, principalmente, aquellos definidos en la misión - accionistas, colaboradores, proveedores, distribuidores, clientes y la sociedad en general.

- Gestionar los riesgos y oportunidades de negocio derivados de la evolución del entorno en el que la empresa desarrolla su actividad, asumiendo el impacto que genera en la sociedad y en el entorno, maximizando los positivos y minimizando los negativos.

- Transmitir el valor social de la empresa, en cada territorio, que concilia el valor de su actividad operativa con el valor de las actividades no lucrativas que se llevan a cabo.

Hipótesis: Si la Responsabilidad Social Corporativa RSC, en el contexto externo e interno se vincula con los procesos del talento humano de una Empresa, gestionándose de manera eficiente y eficaz, entonces, afectará positivamente en el Clima Laboral de dichas Instituciones.

Existen siete ejes en donde la RSC debe trabajar dentro de las organizaciones, Gobernabilidad, Público Interno (talento humano), Medio Ambiente, Proveedores, Mercado, Comunidades y Política Pública. 
ISSN: 2602-8506

Vol. 3, N4., p.98-115, octubre - diciembre, 2019

Las dos primeras (Gobernabilidad, Público Interno) se enfocan especialmente en temas relacionados con los líderes y colaboradores de las empresas, en donde los valores, la transparencia, la ética, así como las condiciones equitativas-justas laborales por parte de los directivos, lograran una producción óptima para la organización y más aún una estabilidad y satisfacción de los colaboradores dentro del Clima laboral de la Empresa. Los otros puntos se refieren al trato y buena relación de la compañía con los protagonistas externos y que son los nuevos socios estratégicos de la compañía.

Los principios expuestos en el Pacto Mundial y en los Convenios de la Organización Internacional del Trabajo (respecto a los Derechos Humanos, eliminación del trabajo forzoso, derecho a la sindicalización colectiva, remuneración equitativa, indistintamente del género ,eliminación de la discriminación por etnia, religión, género, etc., normas de salud y seguridad en el trabajo, erradicación del trabajo infantil, entre otros); sirven, como una guía en el manejo efectivo de la RSC dentro de la organización para que en la misma se pueda apreciar un buen ambiente laboral.

Muchas de las prácticas llevadas a cabo en la aplicación de la RSC no representan un beneficio económico para los colaboradores, si no, se fortalece en el salario emocional, muchos de ellos se convierten en beneficios emocionales que la organización brinda para dar un valor adicional como: bienestar, satisfacción laboral, buen vivir y sentido de pertenencia al colaborador.

De seguro, al momento de realizar un estudio de clima laboral, si se manejan buenas prácticas de RSC internamente de la organización, se podrán evidenciar en los resultados un sentido de pertenencia por parte de los colaboradores generando compromiso, mayor productividad, rentabilidad, incremento del valor de mercado de la organización, reducción de costos, mejora la capacidad para atraer, retener y motivar a los mejores talentos, se fortalece la lealtad y confianza de los trabajadores, reduce el absentismo, ausentismo y se consolida la reputación interna y externa de la Empresa.

Un programa de RSC muy bien estructurado, canalizado, comprometido, socializado y puesto en marcha, será exitoso, considerando su "puesta en marcha" primeramente "casa adentro" de las compañías, privilegiando su talento humano y su entorno laboral satisfactorio. 
Para que la RSC tenga un gran impacto dentro de una organización, es necesario gestionar los siguientes pasos:

1. Diagnosticar la composición del Talento que existe en las Organizaciones.

2. Identificar el talento que se requiere para cada Área Estructural o Funcional de la

Organización, así como para cada puesto de trabajo.

3. Ubicar el Talento "adecuado" en relación con los requerimientos para que generen impactos.

4. Estimular los buenos procesos de selección, la motivación, desarrollo y la retención del Talento.

5. Controlar el desempeño del Talento con un método adecuado e idóneo.

Para gestionar la RSC se deberá confeccionar un Plan de Responsabilidad Social Corporativa, que incluya una Memoria de Sostenibilidad, en la que se refleje sus actuaciones como acción sostenible. Debemos entonces tener en las Gerencias de Talento Humano un enfoque de gestión fundamentado en apoyar a viabilizar el plan estratégico, valores, la visión y misión institucional a través de la efectiva y oportuna aplicación de los procesos de incorporación, permanencia y desvinculación de personal desde una perspectiva de alineamiento estratégico y orientación a los grupos de interés. Este nuevo enfoque supondría que todas las actividades del área deberían estar orientadas a cómo apoyar a que la estrategia se cumpla y en cómo podemos atender las expectativas de nuestros socios principales sean estos internos o externos (Paredes, EL Portal del Capital Humano, 2018).

Las principales responsabilidades éticas de la empresa con los trabajadores y la comunidad son:

- Servir a la sociedad con productos útiles y en condiciones justas.

- Crear riqueza de la manera más eficaz posible.

- Respetar los derechos humanos con unas condiciones de trabajo dignas que favorezcan la seguridad y salud laboral y el desarrollo humano y profesional de los trabajadores.

- Procurar la continuidad de la empresa y, si es posible, lograr un crecimiento razonable.

- Respetar el medio, evitando en lo posible cualquier tipo de contaminación, minimizando la generación de residuos y racionalizando el uso de los recursos naturales y energéticos.

- Cumplir con rigor las leyes, reglamentos, normas y costumbres, respetando los legítimos contratos y compromisos adquiridos. 
- Procurar la distribución equitativa de la riqueza generada.

- Seguimiento del cumplimiento de la legislación por parte de la empresa.

- Mantenimiento de la ética empresarial y lucha contra la corrupción.

- Supervisión de las condiciones laborales y de salud de los/as trabajadores/as.

- Seguimiento de la gestión de los recursos y los residuos.

- Revisión de la eficiencia energética de la empresa.

- Correcto uso del agua.

- Lucha contra el cambio climático.

- Evaluación de riesgos ambientales y sociales.

- Supervisión de la adecuación de la cadena de suministro.

- Diseño e implementación de estrategias de asociación y colaboración de la empresa.

- Implicar a los consumidores, comunidades locales y resto de la sociedad.

- Implicar a los empleados en las buenas prácticas de Responsabilidad Social Corporativa.

- Marketing y construcción de la reputación corporativa.

\section{Decálogo del Responsable de Talento Humano:}

Conjunto de reglas que se consideran básicas para una actividad de responsabilidad Social Corporativa.

- Busca soluciones a los conflictos del talento humano a través del análisis.

- Procura evitar conflictos interpersonales.

- Mantiene siempre las relaciones interpersonales óptimas.

- Logra acuerdos y soluciones rápidamente.

- Para entender el problema genera "empatía" con las personas.

- Demanda tiempo para conocer los problemas personales, laborales de sus colaboradores.

- Crea canales efectivos de comunicación de doble vía.

- Sabe escuchar, no solo oye.

- Tiene un alto nivel de paciencia y tolerancia.

- Busca constantemente las soluciones a los problemas que se le presentan.

\section{Metodología}

Explicación descriptiva (cuantitativa, cualitativa) investigación de campo, de un caso práctico realizado en una Compañía Industrial Comercial en el Ecuador. Luego de haber presentado a la 
ISSN: 2602-8506

Vol. 3, N4., p.98-115, octubre - diciembre, 2019

Dirección de la Empresa los resultados del análisis de los factores logrados en la Encuesta de Clima Laboral de dicha compañía en el año 2018, se concluyó que el mejoramiento continuo del Clima Laboral en la Empresa en sus diferentes procesos es un "Factor Diferenciador" de la Compañía con la competencia y que genera un gran impacto en la Responsabilidad Social Corporativa. De esta manera, evidenciamos que las buenas prácticas en RSC afecta positivamente en los resultados de medición del Clima Laboral en la compañía objeto de este estudio.

\section{Porcentaje de participación:}

La encuesta de Medición de Clima Laboral alcanzó una representatividad del 98\% tomando el número de colaboradores de la Empresa como referencia. Este alto porcentaje alcanzado demuestra con confiabilidad y validez el sentir de la gente que trabaja en esta Compañía.

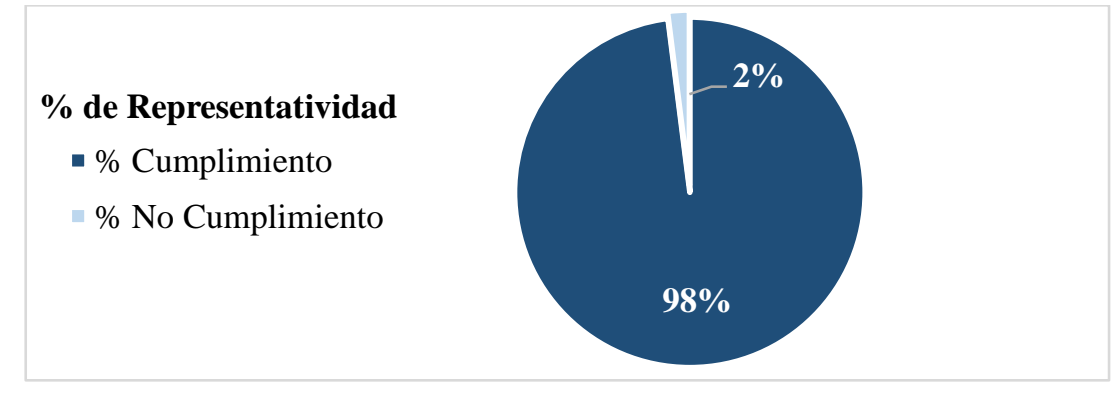

Figura 1. Porcentaje de participación

Fuente: Encuesta de Medición de Clima Laboral (2018).

\section{Metodología Aplicada:}

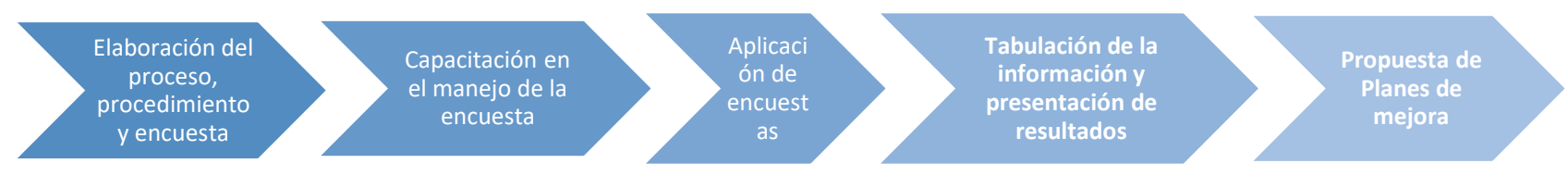

Figura 2. Metodología aplicada al proyecto

Fuente: autores 2018.

- Se elaboró el proceso, procedimiento, políticas y la encuesta de Clima Laboral.

- Una vez aprobado el procedimiento por Gerencia, se procedió a realizar capacitaciones personalizadas para cada uno de los colaboradores de la Empresa para que se instruyan en el uso y llenado de la encuesta. 
ISSN: 2602-8506

www.visionariodigital.org

Vol. 3, N4., p.98-115, octubre - diciembre, 2019

- Aplicación de las encuestas de al menos 90\% de los colaboradores de la Empresa.

- Talento Humano tabula la información bruta para organizarla en cuadros comparativos y presentarlas a Gerencia y el equipo de líderes de la organización, para que éstos a su vez compartan con sus equipos de trabajo y de esta manera para que toda la Organización se comprometa en su "mejora continua".

- Conforme al análisis e interpretación de datos se procederá a realizar planes de mejora con cada uno de los líderes de área y sus equipos de trabajo.

\section{Resultados}

\section{Resultados de satisfacción por factor}

La siguiente gráfica nos indica el promedio de satisfacción de cada factor de la encuesta, las mismas que están conformadas por preguntas que serán analizadas más adelante.

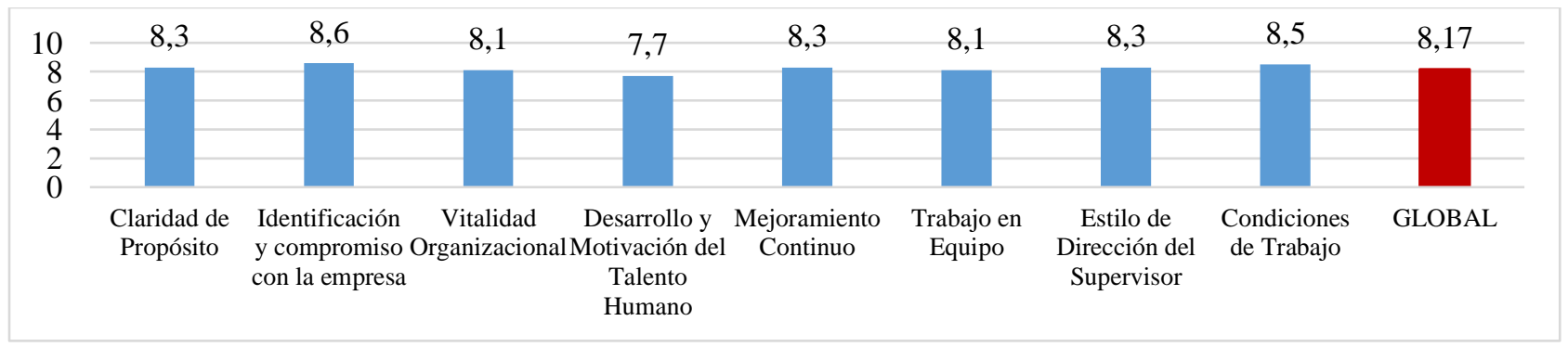

Figura 3. Resultado de satisfacción por dimensión

Fuente: Encuesta de Medición de Clima Laboral (2018).

\section{Resultados de satisfacción por variable}

La siguiente gráfica nos indica el promedio de satisfacción de cada variable de la encuesta: Área / Edad / Género / Tiempo en la empresa / Lugar de Trabajo.

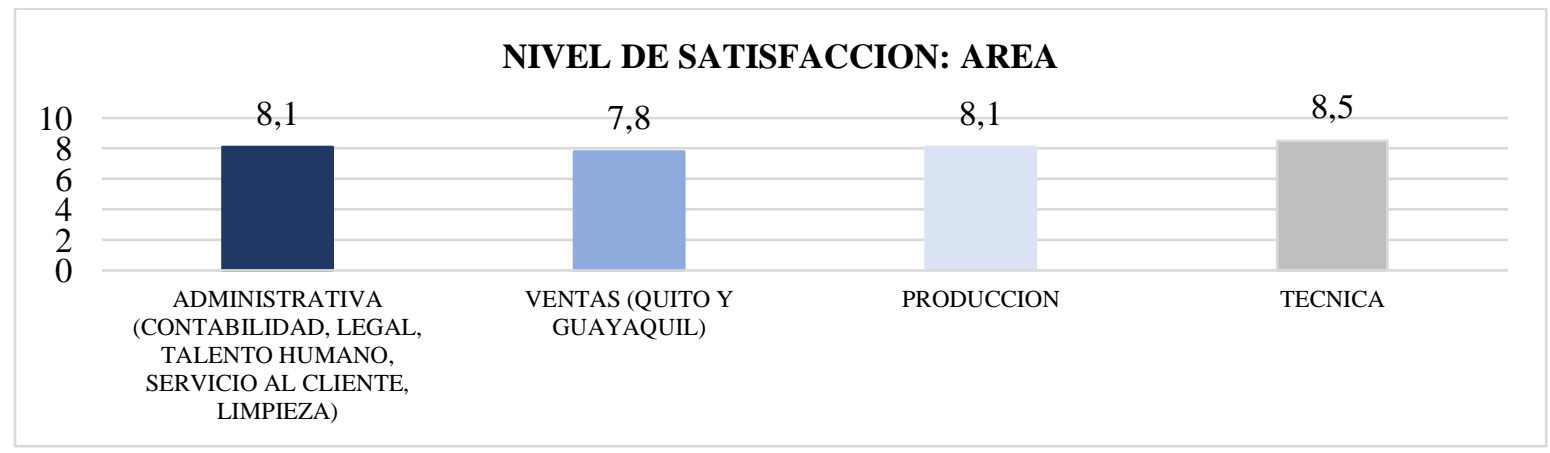

Figura 4. Nivel de satisfacción por área

Fuente: Encuesta de Medición de Clima Laboral (2018). 
ISSN: 2602-8506

Vol. 3, N4., p.98-115, octubre - diciembre, 2019

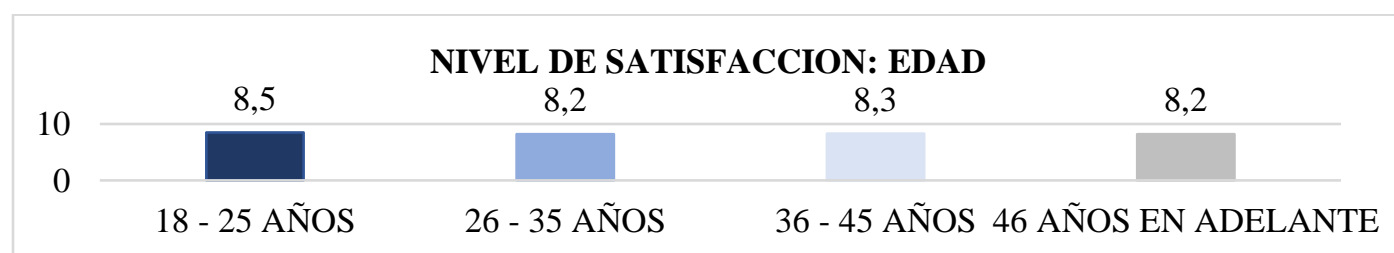

Figura 5. Nivel de satisfacción por edad

Fuente: Encuesta de Medición de Clima Laboral (2018).

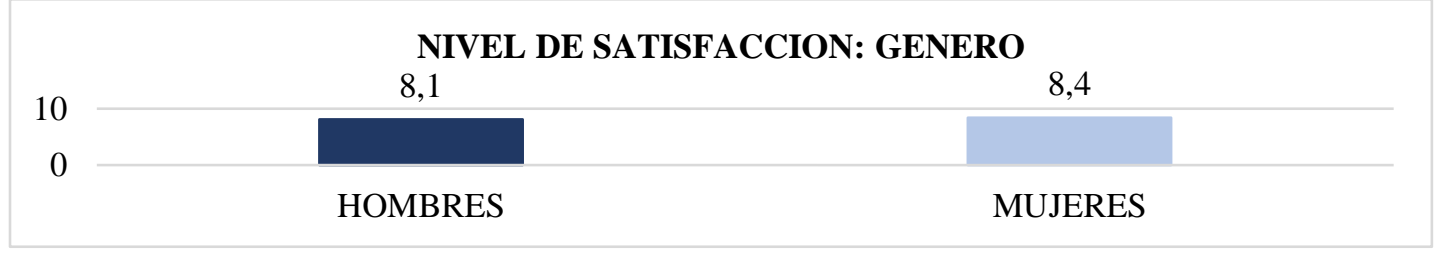

Figura 6. Nivel de satisfacción por género

Fuente: Encuesta de Medición de Clima Laboral (2018).

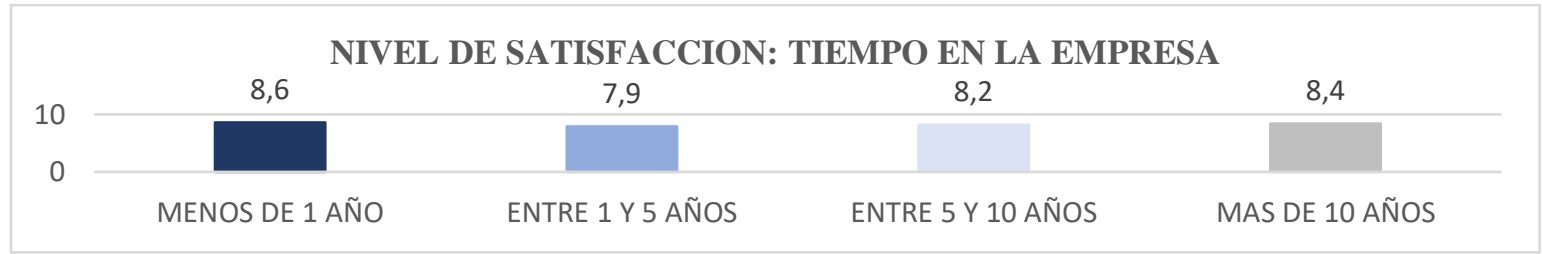

Figura 7. Nivel de satisfacción por tiempo en la empresa

Fuente: Encuesta de Medición de Clima Laboral (2018).

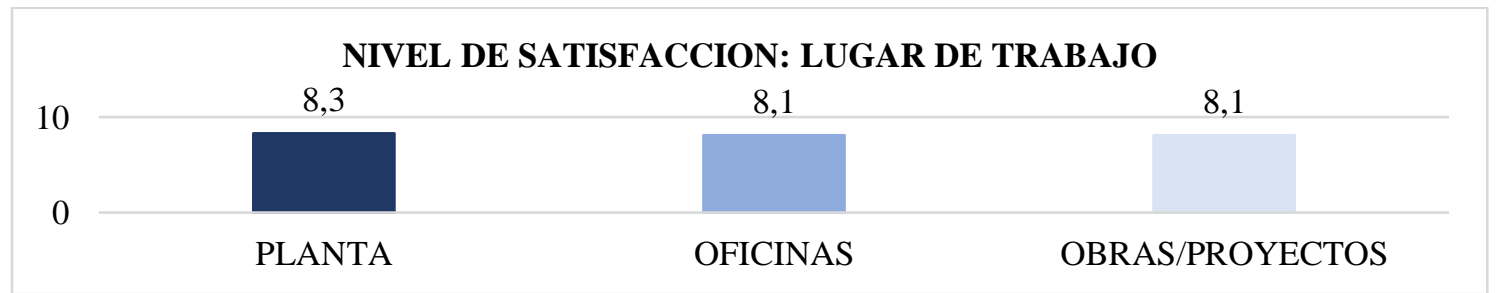

Figura 8. Nivel de satisfacción por lugar de trabajo

Fuente: Encuesta de Medición de Clima Laboral (2018).

\section{Promedio de satisfacción por pregunta}

En el siguiente cuadro se puede evidenciar el promedio de satisfacción alcanzado en cada una de las preguntas, las mismas que están agrupadas por factores. Las preguntas que alcanzaron promedios menores a 7,6 están resaltadas para localizarlas más fácilmente: 
ISSN: 2602-8506

www.visionariodigital.org

Vol. 3, N4., p.98-115, octubre - diciembre, 2019

Tabla 1. Dimensión: claridad de propósito

\begin{tabular}{clc}
\hline DIMENSION & \multicolumn{1}{c}{ PREGUNTAS } & $\begin{array}{c}\text { PROMEDIO DE } \\
\text { SATISFACCION }\end{array}$ \\
\hline $\begin{array}{c}\text { CLARIDAD } \\
\text { DE }\end{array}$ & $\begin{array}{l}\text { Conozco las competencias que deben tener las personas que trabajan en mi } \\
\text { PROPOSITO }\end{array}$ & $\mathbf{7 . 3}$ \\
& $\begin{array}{l}\text { compañía } \\
\text { En mi área de trabajo existe claridad sobre las funciones que cada empleado } \\
\text { debe desempeñar }\end{array}$ & $\mathbf{8 . 3}$ \\
$\mathbf{8 , 3}$ & $\begin{array}{l}\text { Tengo claro cómo se mide el cumplimiento de mis resultados } \\
\text { En mi área de trabajo, todas las tareas que se realizan contribuyen a los } \\
\text { resultados de la compañía }\end{array}$ & $\mathbf{7 . 9}$ \\
& Tengo claro cómo mi trabajo contribuye a los resultados de la compañía & $\mathbf{9 . 0}$ \\
\hline
\end{tabular}

Fuente: Encuesta de Medición de Clima Laboral (2018).

Tabla 2. Dimensión: identificación y compromiso con la empresa

\begin{tabular}{|c|c|c|}
\hline DIMENSION & PREGUNTAS & $\begin{array}{l}\text { PROMEDIO DE } \\
\text { SATISFACCION }\end{array}$ \\
\hline$\underset{Y}{\text { IDENTIFICACION }}$ & $\begin{array}{l}\text { Las personas de mi área cuidan y dan el uso adecuado a los recursos } \\
\text { disponibles (tiempo, dinero, información y bienes de la compañía) }\end{array}$ & 8.2 \\
\hline \multirow[t]{2}{*}{$\begin{array}{l}\text { COMPROMISO } \\
\text { CON LA EMPRESA }\end{array}$} & $\begin{array}{l}\text { Las personas de mi área están comprometidas con la compañía y trabajan } \\
\text { con energía }\end{array}$ & 8.6 \\
\hline & $\begin{array}{l}\text { Las personas de mi área están dispuestas a contribuir al máximo en su } \\
\text { trabajo }\end{array}$ & 8.5 \\
\hline \multirow[t]{5}{*}{8,6} & La compañía cumple mis expectativas como empresa para trabajar & 8.4 \\
\hline & $\begin{array}{l}\text { Mi trabajo tiene un significado especial, éste no es solamente un trabajo } \\
\text { más en mi vida }\end{array}$ & 8.9 \\
\hline & Considero que ésta es una empresa atractiva para trabajar & 8.6 \\
\hline & Me siento orgulloso de trabajar aquí & 8.9 \\
\hline & Me gustaría trabajar en esta compañía muchos años más & 8.9 \\
\hline
\end{tabular}

Fuente: Encuesta de Medición de Clima Laboral (2018).

Tabla 3. Dimensión: vitalidad organizacional

\begin{tabular}{clc}
\hline DIMENSION & \multicolumn{1}{c}{ PREGUNTAS } & $\begin{array}{c}\text { PROMEDIO DE } \\
\text { SATISFACCION }\end{array}$ \\
\hline $\begin{array}{c}\text { VITALIDAD } \\
\text { ORGANIZACIONAL }\end{array}$ & La presión por el logro de resultados es manejable & $\mathbf{8 . 5}$ \\
\cline { 2 - 3 } & La estructura de mi área facilita el logro de resultados & $\mathbf{8 . 3}$ \\
$\mathbf{8 , 1}$ & En esta empresa las decisiones se toman con agilidad & $\mathbf{7 . 7}$ \\
& Esta empresa responde con rapidez a los cambios & $\mathbf{7 . 4}$ \\
& Esta empresa es percibida como líder de mercado & $\mathbf{8 . 0}$ \\
& El área en la que trabajo se preocupa por crecer al ritmo de la & $\mathbf{8 . 7}$ \\
\hline
\end{tabular}

Fuente: Encuesta de Medición de Clima Laboral (2018). 
ISSN: 2602-8506

www.visionariodigital.org

Vol. 3, N4., p.98-115, octubre - diciembre, 2019

Tabla 4. Dimensión: desarrollo y motivación del talento humano

\begin{tabular}{|c|c|c|}
\hline DIMENSION & PREGUNTAS & $\begin{array}{l}\text { PROMEDIO DE } \\
\text { SATISFACCION }\end{array}$ \\
\hline \multirow{13}{*}{$\begin{array}{c}\text { DESARROLLO Y } \\
\text { MOTIVACION } \\
\text { DEL TALENTO } \\
\text { HUMANO }\end{array}$} & Siento que se da el mismo trato a todos los empleados & 7.1 \\
\hline & En mi área se promueven espacios de integración & 6.9 \\
\hline & $\begin{array}{l}\text { Siento que soy importante para la compañía y que ésta se preocupa } \\
\text { por mi bienestar }\end{array}$ & 7.9 \\
\hline & $\begin{array}{l}\text { La compañía me brinda el entrenamiento requerido para realizar } \\
\text { mejor mi trabajo }\end{array}$ & 7.4 \\
\hline & $\begin{array}{l}\text { Además de mi supervisor, existen otras instancias o personas a las } \\
\text { que puedo acudir cuando tengo inquietudes }\end{array}$ & 8.9 \\
\hline & $\begin{array}{l}\text { En mi área de trabajo encuentro retos que permiten mi desarrollo } \\
\text { personal y profesional }\end{array}$ & 8.6 \\
\hline & $\begin{array}{l}\text { La inducción que reciben las personas al ingresar a la compañía es } \\
\text { adecuada }\end{array}$ & 7.2 \\
\hline & $\begin{array}{l}\text { La empresa realiza celebraciones y actividades de integración y } \\
\text { recreación de alto impacto }\end{array}$ & 6.7 \\
\hline & $\begin{array}{l}\text { La empresa demuestra interés en mí como persona y no solo como } \\
\text { trabajador }\end{array}$ & 7.9 \\
\hline & En el último año, participó en cursos de capacitación & 6.7 \\
\hline & $\begin{array}{l}\text { La empresa brinda estabilidad laboral en la medida que se tenga un } \\
\text { desempeño adecuado }\end{array}$ & 8.8 \\
\hline & $\begin{array}{l}\text { En la empresa existen espacios o mecanismos para transmitir mis } \\
\text { ideas y ser escuchado }\end{array}$ & 7.7 \\
\hline & $\begin{array}{l}\text { Cuando las personas recién se vinculan a la compañía se les hace } \\
\text { sentir rápidamente "en casa" }\end{array}$ & 8.2 \\
\hline
\end{tabular}

Fuente: Encuesta de Medición de Clima Laboral (2018).

Tabla 5. Dimensión: mejoramiento continuo

\begin{tabular}{clc}
\hline \multicolumn{1}{c}{ DIMENSION } & \multicolumn{1}{c}{ PREGUNTAS } & $\begin{array}{c}\text { PROMEDIO DE } \\
\text { SATISFACCION }\end{array}$ \\
\hline $\begin{array}{c}\text { MEJORAMIENTO } \\
\text { CONTINUO }\end{array}$ & $\begin{array}{l}\text { En mi área somos disciplinados en el cumplimiento de los } \\
\text { procedimientos, compromisos y normas }\end{array}$ & $\mathbf{8 . 4}$ \\
$\mathbf{8 , 3}$ En mi área los problemas son analizados a fondo & $\begin{array}{l}\text { En mi área existen procedimientos que facilitan el logro de } \\
\text { resultados }\end{array}$ & $\mathbf{8 . 1}$ \\
& $\begin{array}{l}\text { En mi área de trabajo nos preocupamos por mejorar procesos, } \\
\text { herramientas y formas de trabajo } \\
\text { Mis ideas y opiniones son tenidas en cuenta en la solución de } \\
\text { los problemas de mi área o sección } \\
\text { Conozco los procedimientos que son importantes para el } \\
\text { desempeño adecuado de mi trabajo }\end{array}$ & $\mathbf{8 . 5}$ \\
\hline
\end{tabular}

Fuente: Encuesta de Medición de Clima Laboral (2018). 
ISSN: 2602-8506

www.visionariodigital.org

Vol. 3, N4., p.98-115, octubre - diciembre, 2019

Tabla 6. Dimensión: Estilo de dirección del supervisor

\begin{tabular}{|c|c|c|}
\hline DIMENSION & PREGUNTAS & $\begin{array}{l}\text { PROMEDIO DE } \\
\text { SATISFACCION }\end{array}$ \\
\hline \multirow[t]{2}{*}{$\begin{array}{c}\text { ESTILO DE } \\
\text { DIRECCION } \\
\text { DEL } \\
\text { SUPERVISOR }\end{array}$} & Mi supervisor practica lo que predica & DA TIDF ACTON \\
\hline & Mi supervisor me da reconocimiento cuando hago algo sobresaliente & 7.6 \\
\hline \multirow[t]{15}{*}{8,3} & $\begin{array}{l}\text { Cuando se cometen errores mi supervisor tiende a corregirlos de forma } \\
\text { positiva }\end{array}$ & 7.8 \\
\hline & $\begin{array}{l}\text { Me entero de las cosas por una comunicación oficial de mi supervisor } \\
\text { más que por comentarios informales }\end{array}$ & 7.4 \\
\hline & Mi supervisor ayuda a generar un buen ambiente de trabajo & 8.4 \\
\hline & El seguimiento que mi supervisor realiza a mi trabajo es adecuado & 8.5 \\
\hline & $\begin{array}{l}\text { Acuerdo con mi supervisor los objetivos de trabajo y las actividades } \\
\text { para lograrlos }\end{array}$ & 8.7 \\
\hline & $\begin{array}{l}\text { La orientación que brinda mi supervisor para realizar el trabajo es } \\
\text { adecuada }\end{array}$ & 8.5 \\
\hline & Mi supervisor evalúa con objetividad mi trabajo & 8.2 \\
\hline & Mi supervisor me reta (incentiva) y me lleva a que aprenda día a día & 8.0 \\
\hline & Mi supervisor promueve la participación y el aporte de nuevas ideas & 8.1 \\
\hline & $\begin{array}{l}\text { Cuando hago una pregunta a mi supervisor soy escuchado y recibo una } \\
\text { respuesta }\end{array}$ & 8.6 \\
\hline & $\begin{array}{l}\text { Cuando tengo que atender una necesidad personal encuentro una actitud } \\
\text { receptiva por parte de mi supervisor }\end{array}$ & 8.8 \\
\hline & Participó usted en la toma de decisiones en su área de trabajo & 8.1 \\
\hline & $\begin{array}{l}\text { En general, está de acuerdo con la forma como su supervisor gestiona el } \\
\text { departamento }\end{array}$ & 8.6 \\
\hline & El trato que recibo de mi supervisor es respetuoso & 9.4 \\
\hline & $\begin{array}{l}\text { Confía en la capacidad de su supervisor para llevar a cabo las metas del } \\
\text { departamento }\end{array}$ & 9.1 \\
\hline
\end{tabular}

Fuente: Encuesta de Medición de Clima Laboral (2018).

Tabla 7. Dimensión: condiciones de trabajo

\begin{tabular}{clc}
\hline \multirow{2}{*}{ DIMENSION } & \multicolumn{1}{c}{ PREGUNTAS } & \begin{tabular}{c}
\multicolumn{1}{c}{ PROMEDIO DE } \\
SATISFACCION
\end{tabular} \\
\hline $\begin{array}{c}\text { CONDICIONES } \\
\text { DE TRABAJO }\end{array}$ & $\begin{array}{l}\text { La empresa proporciona las condiciones de trabajo suficientes y } \\
\text { necesarias para proteger la salud y seguridad de su personal }\end{array}$ & $\mathbf{8 . 1}$ \\
& Mi horario de trabajo es adecuado & $\mathbf{8 . 6}$ \\
$\mathbf{8 , 5}$ & La compañía brinda las herramientas requeridas para realizar bien & $\mathbf{8 . 3}$ \\
& el trabajo & $\mathbf{8 . 4}$ \\
& Las instalaciones físicas son agradables & $\mathbf{8 . 7}$ \\
& La empresa me paga puntualmente el salario & $\mathbf{8 . 7}$ \\
\hline
\end{tabular}

Fuente: Encuesta de Medición de Clima Laboral (2018). 
ISSN: 2602-8506

www.visionariodigital.org

Vol. 3, N4., p.98-115, octubre - diciembre, 2019

Tabla 8. Preguntas de mayor nivel de satisfacción

PREGUNTAS CON MAYOR NIVEL DE SATISFACCION

PROMEDIO DE SATISFACCION

El trato que recibo de mi supervisor es respetuoso

9.4

Tengo claro cómo mi trabajo contribuye a los resultados de la compañía

Confía en la capacidad de su supervisor para llevar a cabo las metas del departamento

En mi área de trabajo, todas las tareas que se realizan contribuyen a los resultados de la compañía

Me siento orgulloso de trabajar aquí

Me gustaría trabajar en esta compañía muchos años más

Mi trabajo tiene un significado especial, éste no es solamente un trabajo más en mi vida

Además de mi supervisor, existen otras instancias o personas a las que puedo acudir cuando tengo inquietudes

Conozco los procedimientos que son importantes para el desempeño adecuado de mi trabajo

Cuando tengo que atender una necesidad personal encuentro una actitud receptiva por parte de mi supervisor

La empresa brinda estabilidad laboral en la medida que se tenga un desempeño adecuado

9.1

9.0

8.9

8.9

8.8

Fuente: Encuesta de Medición de Clima Laboral (2018).

Tabla 9. preguntas con el menor nivel de satisfacción

\section{PREGUNTAS CON MENOR NIVEL DE SATISFACCION}

Mi supervisor me da reconocimiento cuando hago algo sobresaliente

Recibo oportunamente la información que requiero de otras áreas para realizar mi trabajo

Esta empresa responde con rapidez a los cambios

La compañía me brinda el entrenamiento requerido para realizar mejor mi trabajo

Me entero de las cosas por una comunicación oficial de mi supervisor más que por comentarios informales

Conozco las competencias que deben tener las personas que trabajan en mi compañía

La inducción que reciben las personas al ingresar a la compañía es adecuada

Siento que se da el mismo trato a todos los empleados

En mi área se promueven espacios de integración

En el último año, participó en cursos de capacitación

La empresa realiza celebraciones y actividades de integración y recreación de alto impacto
PROMEDIO DE SATISFACCION

Fuente: Encuesta de Medición de Clima Laboral (2018). 


\section{Oportunidades de "mejora continua" para trabajar en esta compañía:}

Los líderes de la compañía deben involucrar más a sus equipos de trabajo en la toma de decisiones y reconocer sus logros. Además, debe existir una comunicación formal de todo lo que sucede en la compañía.

Fortalecer la comunicación interdepartamental con el propósito de realizar efectivos y eficientes procesos de trabajo en equipo evitando atrasos, desperdicios y reprocesos.

Efectuar eventos de integración con la finalidad de disfrutar otros espacios lúdicos de recreación fortaleciendo las relaciones interpersonales entre todos los niveles de la compañía.

Mejorar los procesos de capacitación, educación, entrenamiento en todos los niveles de la organización.

Desarrollo humano de manera sostenida fortalecerá sus procesos dentro de la compañía tales como: Competencias del personal, capacitación, planes de carrera, evaluación al desempeño, inducción, buenas prácticas legales laborales con el propósito de fortalecer al Talento Humano en la adaptación a los nuevos cambios que exige la compañía.

\section{Conclusiones}

Es importante que la empresa empiece con un proceso de diseño y documentación de las prácticas de RSC que desarrolla, a fin de medir los efectos que están teniendo en relación con la satisfacción de sus colaboradores. De esta manera, se establecen mejoras o correctivos que hacen de las actividades orientadas a los trabajadores y sus familias procesos efectivos de mejoramiento en la calidad de vida.

Para futuras investigaciones resulta importante analizar el plan estratégico de las organizaciones objeto de estudio y la forma como la RSC se vincula como estrategia de gestión vista e implementada en algunos campos desde la gestión humana.

El papel que representa el área de gestión humana en el diseño y aplicación de prácticas de responsabilidad social empresarial es protagónico, ya que todas estas actividades se enfocan a los trabajadores y a sus familias, los cuales representan el principal objetivo del área.

El área de Gestión de talento humano se vincula directamente al direccionamiento estratégico de la empresa, lo que permite realizar una alineación efectiva entre las metas organizacionales y la RSC y garantiza, en cierta medida, la aplicación efectiva de dichas prácticas. 
ISSN: 2602-8506

Vol. 3, N4., p.98-115, octubre - diciembre, 2019

Podemos afirmar que hoy esté aceptada la concepción de que, además de producir bienes y servicios, para asegurar su estabilidad, continuidad y crecimiento las empresas deben cumplir objetivos sociales. Ello no significa que deban encargarse de resolver problemas comunitarios puntuales, sino que responderán como instrumentos para satisfacer las necesidades del talento humano en las Organizaciones.

Todas las empresas pueden aprovechar el "ganar-ganar" que abre la gestión del talento humano: ganan los colaboradores al disfrutar buenas condiciones de trabajo y oportunidades de superación; gana la empresa al tener un personal motivado y más productivo; y gana la sociedad al extenderse las prácticas de trabajo digno y estable que contribuyen al desarrollo.

Para conseguir estos cambios, es indispensable la presencia de líderes con nuevos estilos y enfoques de dirección, que con su orientación puedan alcanzar efectividad en las personas y de la empresa bajo una perspectiva integral y que también obtengan una alta productividad en la organización.

\section{Recomendaciones}

Los departamentos de Talento Humano y el de RSC deben contar con el apoyo de los altos directivos, generando confianza con los grupos de interés.

Incentivos alineados con la RSC mediante este método se consigue integrar a este departamento como parte de los procesos y estructuras de la empresa de un modo efectivo.

Gestión adecuada de los proveedores y clientes.

Contar con el presupuesto necesario para el departamento de RSC y su gestión.

Crear la RSC desde el interior de la empresa.

\section{Referencia bibliográfica}

Armando, C. y Cejas, M. (2009) Responsabilidad Social: factor clave en la gestión de recursos humanos en las organizaciones en el siglo XXI.

Barrera, J; López, F. y Romero, P. (2011). El valor interno de la RSC: implicaciones en la gestión de RRHH y el clima laboral de la empresa. 
ISSN: 2602-8506

Vol. 3, N4., p.98-115, octubre - diciembre, 2019

Carroll, Archie, y Kareem Shabana. 2010. "The Business Case for Corporate Social Responsibility: A Review of Concepts, Research and Practice”. International Journal of Management Reviews 12, No. 1: 85-105.

Celis, J. (13 de febrero de 2017). La Motivación y el éxito personal. Obtenido de http://www.sebascelis.com/motivacion-y-exito/?sub=1\&item=motivacion

Díaz, F, et al. (2014). Competitividad y responsabilidad social corporativa. INCEPTUM Revista de Investigación en Ciencias de la Administración, 3(5), 127-144.

Duarte, et al. (2014). Tell me yoursociallyresponsiblepractices, iwilltellyouhowattractiveforrecruitmentyou are!

Theimpactofperceivedcsronorganizationalattractiveness.Tékhne, 12, Supplement 1, 2229. doi: http://dx.doi.org/10.1016/j.tekhne.2015.01.004

Escat, M. (15 de febrero de 2017). EQUIPO DE TRABAJO Y TRABAJO EN EQUIPO. Obtenido de http://www.leonismoargentino.com.ar/INST219.htm

Hernández, G. et al (2011). Papel de gestión humana en el cumplimiento de la responsabilidad social empresarial. Estudios Gerenciales, 27(118), 163-188. doi: http://dx.doi.org/10.1016/S0123-5923(11)70151-6

Lizcano, J. (2004) ¿Qué es Responsabilidad Social Corporativa?, AECA: España.

Makower, B., (1995).No se puede conducir una empresa saludable en una sociedad enferma por тисho tiempo.

Odriozola, B. (13 de febrero de 2017). Salud Emocional: La Insatisfacción Permanente. Obtenido de https://es-la.facebook.com/notes/xen-pilates/salud-emocional-lainsatisfacci\%C3\%B3n-permanente/10150304996180046

Pérez, J., \& Merino, M. (13 de febrero de 2017). DEFINICION DE MOTIVACIÓN. Obtenido de http://definicion.de/motivacion/ 
ISSN: 2602-8506

Vol. 3, N4., p.98-115, octubre - diciembre, 2019

Prado, A. (2004). Marco Lógico y Conceptual del Modelo de Responsabilidad Social Empresarial para Costa Rica. INCAE: Costa Rica.

Santillán, J. (2014). La Responsabilidad Social Corporativa y los procesos de Talento Humano. Una síntesis de la Tesis de Grado para Ingeniería Comercial. Pontificia Universidad Católica del Ecuador: Quito-Ecuador.

Santillán, W. (2017). Nuevas prácticas para mejorar el clima laboral. Quito: Don Bosco.

Wohlmuth, M. (13 de febrero de 2017). Capacitación, factor para retener al talento de th empresa. Obtenido de http://www.altonivel.com.mx/capacitacion-factor-para-retener-altalento-de-tu-empresa-58550/

\section{Webgrafía:}

https://es.wikipedia.org/wiki/Responsabilidad_social_corporativahttps://www.google.com.ec/s earch?q=buenas+practicas+en+responsabilidad+social+corporativa\&source

https://rrppuft.wordpress.com/2014/07/29/publico-interno-y-externo/

http://concepto.de/comunidad/\#ixzz5770yJg00

https://observatoriorsc.org/la-rsc-que-es/

http://www.foromarketing.com/que-es-la-responsabilidad-corporativa/

http://www.compromisorse.com/rse/2010/11/15/cinco-recomendaciones-para-mejorar-la-rse-

de-tu-empresa/

http://www.alfredoparedesyasociados.com > hr analytics-3

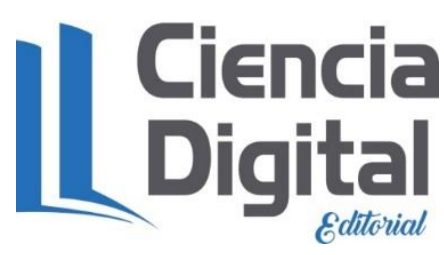




\section{PARA CITAR EL ARTÍCULO INDEXADO}

Santillán Marroquín, W., Paredes García, H., Mena Manotoa, H., \& Santillán Vieira, J. (2019). La responsabilidad social corporativa y el clima laboral. Visionario Digital, 3(4), 98-115. https://doi.org/10.33262/visionariodigital.v3i4.974

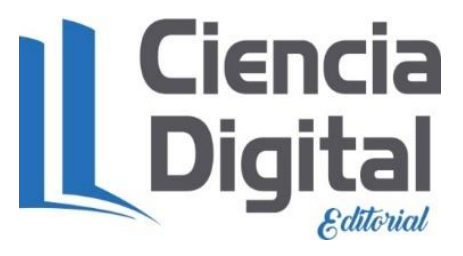

El artículo que se publica es de exclusiva responsabilidad de los autores y no necesariamente reflejan el pensamiento de la Revista Visionario Digital.

El artículo queda en propiedad de la revista y, por tanto, su publicación parcial y/o total en otro medio tiene que ser autorizado por el director de la Revista Visionario Digital.
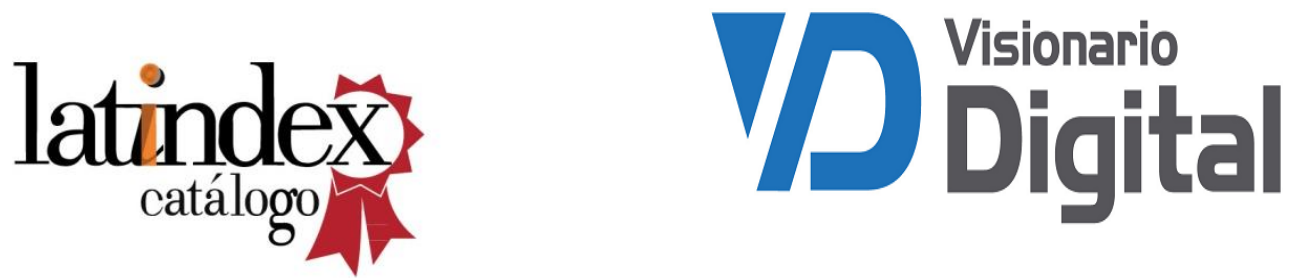